\title{
Location-Aware Cooperative Communications utilizing Linear Network Coding
}

\author{
Hung-Quoc Lai, Ahmed S. Ibrahim*, and K. J. Ray Liu* \\ US Army RDECOM CERDEC, AMSRD-CER-ST-WL-NS, Meyer Center, Ft. Monmouth, NJ 07703, USA. \\ *Department of Electrical and Computer Engineering, University of Maryland, College Park, MD 20742, USA.
}

\begin{abstract}
Cooperative communication can be used to reduce the transmit power of distant mobile units, compared to conventional direct transmission, given the same quality-of-service. However, imposing the constraint of having orthogonal transmission for the source and relays leads to large delay in TDMA systems. For a network of $N$ mobile units, the transmission delay would be $N(N+1) / 2$. In this work, we propose a location-aware cooperation-based scheme that aims to reduce transmit power of distant mobile units while maintaining a low transmission delay. The scheme utilizes a linear network coding protocol, where each mobile unit applies linear network coding to a set of transmit symbols that it has received previously. At the base station, multiuser detection is used to decouple the transmit symbols. Both decode-and-forward and amplify-and-forward cooperative protocols are considered. We show that our proposed scheme achieves a comparable bit-error-rate performance with the conventional cooperation-based TDMA scheme while requiring a delay of $(2 N-1)$ time slots, a substantial reduction in the transmission delay.
\end{abstract}

\section{INTRODUCTION}

In the nature of wireless communications, information from distant mobile units (MUs) to a common base station (BS) requires more transmit power in direct transmission. The additional transmit power is to compensate the large scale fading in order to provide a comparable quality of service (QoS) to that of the close ones. Diversity techniques such as time diversity, frequency diversity, and spatial diversity can result in reduction of transmit power. Among these techniques, spatial diversity achieved by cooperative communication has become recently attractive.

In cooperative communication, MUs in a network acting as relays can process the transmissions overheard from other units. The distributed antennas among the relays are used to provide spatial diversity without the need to use multiple antennas at the source. Various cooperative diversity protocols have been proposed and analyzed in [1]-[6]. Decode-andforward (DAF) and amplify-and-forward (AAF) protocols for cooperative communications are explained in [1]. In DAF protocol, each relay decodes the overheard information from the source, re-encodes it, and then forwards it to the destination. In AAF protocol, each relay simply amplifies the overheard signal and forwards it to the destination. The symbol error rate (SER) for single- and multi-relay DAF protocol was analyzed in [2], [3]. In [4], [5], various relay selection schemes have been proposed that achieve high bandwidth efficiency and full diversity order. Finally, distributed space time codes for DAF and AAF protocols have been proposed and analyzed in [6].

Cooperative communication with its ability to achieve spatial diversity can be used to reduce the transmit power of distant MUs to a common BS in location-aware networks, where locations of nodes are taken into consideration to improve network performance. However, the constraint that source and relay transmissions are over orthogonal channels such as time division multiple access (TDMA) leads to a large transmission delay. For a network of $N$ MUs, the transmission delay would be $N(N+1) / 2$. In this work, we propose a location-aware cooperation-based scheme that aims to reduce transmit power of distant MUs while maintaining a low transmission delay. The scheme uses network coding, where each mobile unit applies linear network coding to a set of transmit symbols that it has received previously to form a unique signal and transmits it to the BS. At the base station, multiuser detection is used to decouple the transmit symbols. Both DAF and AAF protocols in cooperative communications are considered in our proposed scheme. For the validation purpose, the performance analysis is based on BPSK modulation; nevertheless, the extension to general M-PSK and M-QAM can follow directly. We show that our proposed scheme achieves a comparable bit-error-rate (BER) performance with the conventional cooperation-based TDMA scheme while it requires a delay of $(2 N-1)$ time slots, a substantial reduction in transmission delays. Note that only simple detection is required at MUs in our proposed scheme.

This paper is outlined as follows. After this introduction section, our proposed location-aware cooperation-based scheme for DAF and AAF protocols are introduced in Section II. Multiuser detection used in our proposed scheme is presented in Section III for both DAF and AAF protocols. The performance analysis is presented in Section IV to provide a closeform BER expression for DAF protocol and a conditional BER expression for AAF protocol. These expressions then are used in Section $\mathrm{V}$ to obtain analytical and numerical results that validate our proposed scheme. Lastly, we draw some conclusions in Section VI.

\section{LOCATION-AWARE COOPERATION-BASED SCHEME USING LINEAR NETWORK CODING}

We consider an uplink problem of a wireless network consisting of $N$ MUs $U_{1}, U_{2}, \ldots, U_{N}$ and a BS $d$ (destination) as in Figure 1. Without loss of generality, we assume the MUs are numbered in the decreasing order of the distance to the BS. The purpose for $U_{1}, U_{2}, \ldots, U_{N}$ is to transmit a set of symbols $x_{1}, x_{2}, \ldots, x_{N}$ to the BS. Due to large scale fading in direct transmission, $x_{1}$ and $x_{N}$ require the most and the least transmit power, respectively, to provide a comparable QoS.

This proposed scheme aims to provide distributed spatial diversity to the network to reduce the total transmit power while maintaining a low transmission delay. In this scheme, $U_{1}$ with farthest distance seeks help from all hopping terminals, $U_{2}$ with the second farthest distance seeks help from all hopping terminals other than $U_{1}$, and so on. $U_{N}$ with closest 


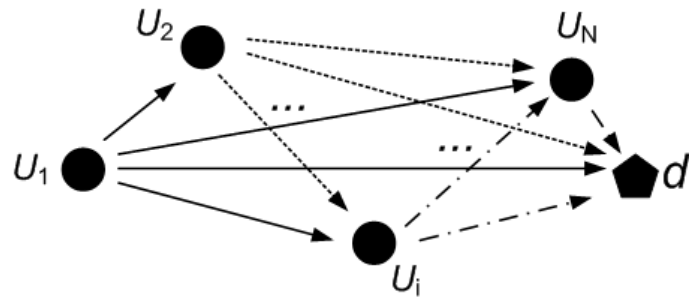

Fig. 1. A wireless network consisting of $N$ mobile units $U_{1}, U_{2}, \ldots, U_{N}$ and a base station $d$.

distance operates under direct transmission mode. We consider both DAF and AAF protocols with the use of network coding at MUs to achieve this objective. Following are system models for the DAF and AAF protocols used in our proposed scheme.

\section{A. DAF Linear Network Coding Protocol}

Each MU $U_{i}$ for $i=2,3, \ldots, N$ is allocated two time slots. In the first time slot, $U_{i}$ applies a linear network coding on the overheard symbols $x_{1}, \ldots, x_{i-1}$ that it has successfully decoded previously to form a linearly coded version of these symbols and transmits it to $d$. In the second time slot, $U_{i}$ transmits its own symbol $x_{i}$ to $U_{i+1}, \ldots, U_{N}$ and $d$. $U_{1}$ has one time slot since it transmits its own symbol only. The total time slots required to transmit a set of $N$ symbols is $(2 N-1)$. In this proposed scheme, $U_{1}$ receives assistance from all MUs while $U_{N}$ receives none; $U_{N}$ operates in direct transmission mode. Figure 2 illustrates the transmission structure of the location-aware cooperative scheme. From this structure, we expect spatial diversity orders of $N, N-1, \ldots, 1$ allocated for $x_{1}, x_{2}, \ldots, x_{N}$, respectively.

To eliminate interference in the linearly coded version of the overheard symbols, each symbol $x_{j}$ is protected by a signature waveform $s_{j}(t)$. The cross-correlation between $s_{j}(t)$ and $s_{i}(t)$ is $\rho_{j i}=<s_{j}(t), s_{i}(t)>$. Let $\widetilde{P}_{i j}^{D}$ ("D" for DAF) be the power allocated at $U_{i}$ in delivery of $x_{j}$. Then [3]

$$
\widetilde{P}_{i j}^{D}=\left\{\begin{array}{ll}
P_{i j} & \text { if } U_{i} \text { decodes } x_{j} \text { correctly } \\
0 & \text { otherwise }
\end{array} .\right.
$$

Power allocation and detection at MUs acting as relays will be described later. Note that the total transmit power to deliver $x_{j}$ is $P_{j}=\sum_{i=j}^{N} P_{i j}$. Also let $h_{u v}$ be a generic channel coefficient representing the channel between any two nodes. $h_{u v}$ is modeled as zero-mean circular symmetric complex Gaussian random variable with variance $\sigma_{u v}^{2}$. Consequently, the channel gain $\left|h_{u v}\right|$ is modeled as Rayleigh random variable [7]. Furthermore, the square of channel gain $\left|h_{u v}\right|^{2}$ is modeled as an exponential random variable with mean $\sigma_{u v}^{2}$, i.e., the probability density function (PDF) of $\left|h_{u v}\right|^{2}$ is [7] $f_{\left|h_{u v}\right|^{2}}\left(\left|h_{u v}\right|^{2}\right)=1 / \sigma_{u v}^{2} \exp \left(-\left|h_{u v}\right|^{2} / \sigma_{u v}^{2}\right) U\left(\left|h_{u v}\right|^{2}\right)$, where $U($.$) is the unit step function.$

Based on these assumptions, the received signals at the destination from $U_{i}$ in the first time slot, $y_{i d r}^{D}(t)$ ("r" for relaying) and in the second time slot, $y_{i d o}^{D}(t)$ ("o" for own) are

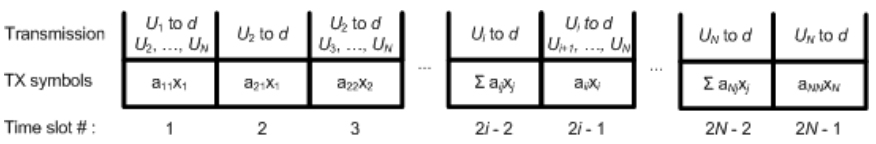

Fig. 2. Transmission structure of location-aware cooperative communication using linear network coding.

$$
\begin{aligned}
& y_{i d r}^{D}(t)=h_{i d} \sum_{j=1}^{i-1} \sqrt{\widetilde{P}_{i j}^{D}} x_{j} s_{j}(t)+n_{i d r}^{D}(t), \\
& y_{i d o}^{D}(t)=\sqrt{P_{i i}} h_{i d} x_{i} s_{i}(t)+n_{i d o}^{D}(t),
\end{aligned}
$$

respectively. Note that the received signal from $U_{1}$ follows (3) with $i=1$. In (2) and (3), $n_{\text {idr }}^{D}(t)$ and $n_{\text {ido }}^{D}(t)$ are modeled as i.i.d. adaptive white Gaussian noise (AWGN) with power spectrum density $N_{0}$. Now we denote $a_{i j}^{D}=\sqrt{\widetilde{P}_{i j}^{D}} h_{i d}$ for $i=1, \ldots, N$ and $j=1, \ldots, i-1$ and $a_{i i}^{D}=\sqrt{P_{i i}} h_{i d}$ as signal coefficients and rewrite (2) and (3) as

$$
\begin{aligned}
& y_{i d r}^{D}(t)=\sum_{j=1}^{i-1} a_{i j}^{D} x_{j} s_{j}(t)+n_{i d r}^{D}(t), \\
& y_{i d o}^{D}(t)=a_{i i}^{D} x_{i} s_{i}(t)+n_{i d o}^{D}(t),
\end{aligned}
$$

respectively.

\section{B. AAF Linear Network Coding Protocol}

The difference between AAF and DAF protocols is that instead of decoding and re-encoding the overheard symbols in the first time slot, $U_{i}$ simply amplifies the overheard signals and forwards a linearly coded version of these signals to $d$. In the second time slot, $U_{i}$ also transmits its own symbol $x_{i}$ to $U_{i+1}, \ldots, U_{N}$ and $d$. Other assumptions for DAF protocol remain the same in AAF protocol.

The received signals at the destination from $U_{i}$ for $i=$ $2, \ldots, N$ in the first time slot, $y_{i d r}^{A}(t)$ ("A" for AAF) and the second time slot, $y_{i d o}^{A}(t)$ are

$$
y_{i d r}^{A}(t)=h_{i d} \sum_{j=1}^{i-1} \frac{\sqrt{P_{i j}}}{\sqrt{P_{j j}\left|h_{j i}\right|^{2}+N_{0}}} y_{j i o}(t)+n_{i d r}^{A}(t),
$$

where

$$
y_{j i o}^{A}(t)=\sqrt{P_{j j}} h_{j i} x_{j} s_{j}(t)+n_{j i o}^{A}(t)
$$

for $j=1, \ldots, i-1$, and

$$
y_{i d o}^{A}(t)=\sqrt{P_{i i}} h_{i d} x_{i} s_{i}(t)+n_{i d o}^{A}(t),
$$

respectively. Note that the received signal from $U_{1}$ follows (8) with $i=1$. In (6) - (8), $n_{i d r}^{A}(t), n_{j i o}^{A}(t)$, and $n_{i d o}^{A}(t)$ are modeled as i.i.d. AWGN with power spectral density $N_{0}$.

Let us take a close look at the signal $y_{i d r}^{A}(t)$. Substituting (7) into (6), we have

$$
y_{i d r}^{A}(t)=h_{i d} \sum_{j=1}^{i-1} \frac{\sqrt{P_{i j} P_{j j}} h_{j i}}{\sqrt{P_{j j}\left|h_{j i}\right|^{2}+N_{0}}} x_{j} s_{j}(t)
$$




$$
\begin{aligned}
& +h_{i d} \sum_{j=1}^{i-1} \frac{\sqrt{P_{i j}}}{\sqrt{P_{j j}\left|h_{j i}\right|^{2}+N_{0}}} n_{j i o}(t)+n_{i d r}^{A}(t) \\
& =\sum_{j=1}^{i-1} a_{i j}^{A} x_{j} s_{j}(t)+\widetilde{n}_{i d r}^{A}(t),
\end{aligned}
$$

where we denote $a_{i j}^{A}=\sqrt{\widetilde{P}_{i j}^{A}} h_{i d}$ as a signal coefficient from $U_{i}$ in association with $x_{j}$ with

$$
\widetilde{P}_{i j}^{A}=\frac{P_{i j} P_{j j}\left|h_{j i}\right|^{2}}{P_{j j}\left|h_{j i}\right|^{2}+N_{0}},
$$

and the noise $\widetilde{n}_{i d r}^{A}(t)$ with power spectral density $N_{0} f_{i}$,

$$
f_{i}=\sum_{j=1}^{i-1} \frac{P_{i j}\left|h_{i d}\right|^{2}}{P_{j j}\left|h_{j i}\right|^{2}+N_{0}}+1
$$

a factor representing the noise amplification impact at $U_{i}$. Likewise, we denote $a_{i i}^{A}=\sqrt{P_{i i}} h_{i d}$ and rewrite (8) as

$$
y_{\text {ido }}^{A}(t)=a_{i i}^{A} x_{i} s_{i}(t)+n_{\text {ido }}^{A}(t) .
$$

\section{A General Form for DAF and AAF Protocols}

We see that DAF and AAF protocols share the same system models with different parameters. In general, the transmit signals from $U_{i}$ in the first and the second time slot are

$$
\begin{aligned}
& y_{i d r}(t)=\sum_{j=1}^{i-1} a_{i j} x_{j} s_{j}(t)+n_{i d r}(t), \\
& y_{i d o}(t)=a_{i i} x_{i} s_{i}(t)+n_{i d o}(t),
\end{aligned}
$$

respectively. In the above equations, $a_{i i}=\sqrt{P_{i i}} h_{i d}, a_{i j}=$ $\sqrt{\widetilde{P}_{i j}} h_{i d}$ for $i=1, \ldots, N$ and $j=1, \ldots, i-1$ where $\widetilde{P}_{i j}$ follows (1) for DAF and (10) for AAF, and the power spectral density of $n_{i d o}(t)$ and $n_{i d r}(t)$ is $N_{0}$ and $N_{i}=N_{0} f_{i}$, respectively, where

$$
f_{i}=\left\{\begin{array}{ll}
1 & \text { for DAF } \\
\sum_{j=1}^{i-1} \frac{P_{i j}\left|h_{i d}\right|^{2}}{P_{j j}\left|h_{j i}\right|^{2}+N_{0}}+1 & \text { for AAF }
\end{array} .\right.
$$

\section{Data Detection at Base Station}

In this section, we use multiuser detection at the BS for our proposed scheme. Data detection is performed by first applying matched-filtering to the received signals with respect to signature waveforms. Then multiuser detection is followed to obtain a set of desired symbols.

\section{A. Matched Filtering}

Given the system models in Section II, the BS receives $N$ direct transmissions in the odd time slots and $(N-1)$ relaying transmissions in the even ones. Matched-filtering with respect to signature waveforms is applied to the received signals to produce a total of $M=\frac{N(N+1)}{2}$ discrete-time signals of the form

$$
y_{i d j}=<y_{i d r}(t), s_{j}(t)>=a_{i j} x_{j}+\sum_{k=1 ; k \neq j}^{i-1} a_{i k} \rho_{j k} x_{k}+n_{i d j},
$$

$$
y_{i d i}=<y_{i d o}(t), s_{i}(t)>=a_{i i} x_{i}+n_{i d i}
$$

for $i=1, \ldots, N$ and $j=1, \ldots, i-1$. In (16) and (17), $n_{i d i} \sim$ $\mathcal{N}\left(0, N_{0}\right)$ and $n_{i d j} \sim \mathcal{N}\left(0, N_{0} f_{i}\right)$ are the AWGN.

Let $\mathbf{y}=\left[y_{1 d 1}, y_{2 d 1}, \ldots, y_{i d j}, \ldots, y_{N d N}\right]^{T}$ be the received signal vector and $\mathbf{R}_{i}=\left\langle\mathbf{s}_{i}, \mathbf{s}_{i}^{T}\right\rangle$ be the cross-correlation matrix where $\mathbf{s}_{i}=\left[s_{1}(t), s_{2}(t), \ldots, s_{i}(t)\right]^{T}$. We can write

$$
\mathbf{y}=\mathbf{R A x}+\mathbf{n},
$$

where $\mathbf{x}=\left[x_{1}, x_{2}, \ldots, x_{N}\right]^{T}, \mathbf{R}=\operatorname{diag}\left\{1, \mathbf{R}_{1}, 1, \ldots\right.$, $\left.\mathbf{R}_{i}, 1, \ldots, \mathbf{R}_{N-1}, 1\right\}_{M \times M}$, and

$$
\mathbf{A}=\left[\begin{array}{lc}
\operatorname{diag}\left(a_{11}\right) & \mathbf{0}_{1 \times(N-1)} \\
\operatorname{diag}\left(a_{21}, a_{22}\right) & \mathbf{0}_{2 \times(N-2)} \\
\ddots & \vdots \\
\operatorname{diag}\left(a_{i 1}, \ldots, a_{i j}, \ldots, a_{i i}\right) & \mathbf{0}_{i \times(N-i)} \\
\ddots & \vdots \\
\operatorname{diag}\left(a_{N 1}, \ldots, a_{N j}, \ldots, a_{N N}\right) &
\end{array}\right]_{M \times N}
$$

Also in (18), $\mathbf{n} \sim \mathcal{N}\left(\mathbf{0}, N_{0} \widetilde{\mathbf{R}}\right)$ where $\widetilde{\mathbf{R}}=\operatorname{diag}\left\{1, \widetilde{\mathbf{R}}_{1}, 1, \ldots\right.$, $\left.\widetilde{\mathbf{R}}_{i-1}, 1, \ldots, \widetilde{\mathbf{R}}_{N-1}, 1\right\}$ with $\widetilde{\mathbf{R}}_{i-1}=f_{i} \mathbf{R}_{i-1}$. If we let $\mathbf{F}=$ $\operatorname{diag}\{1, f_{2}, 1, \ldots, \underbrace{f_{i}, \ldots, f_{i}}_{(\mathrm{i}-1) \text { times }}, 1, \ldots, 1\}$, then $\widetilde{\mathbf{R}}=\mathbf{F R}$.

\section{B. Multiuser Detection Scheme}

Assume $\mathbf{R}_{i}$ is invertible with the invert matrix $\mathbf{R}_{i}^{-1}$. Then the inverse of $\mathbf{R}$ exists with $\mathbf{R}^{-1}=$ $\operatorname{diag}\left\{1, \mathbf{R}_{1}^{-1}, 1, \ldots, \mathbf{R}_{i-1}^{-1}, 1, \ldots, \mathbf{R}_{N-1}^{-1}, 1\right\}$. Multiuser detection is applied to the received signal vector in two steps. First the vector $\mathbf{y}$ is pre-multiplied with the inverse $\mathbf{R}^{-1}$ to obtain

$$
\widetilde{\mathbf{y}}=\mathbf{R}^{-1} \mathbf{y}=\mathbf{A x}+\widetilde{\mathbf{n}},
$$

where $\widetilde{\mathbf{n}} \sim \mathcal{N}\left(\mathbf{0}, N_{0} \mathbf{R}^{-1} \mathbf{F}\right)$. Then $\widetilde{\mathbf{y}}$ is grouped into signal vectors $\mathbf{y}_{j}$ in association with the desired symbols $x_{j}$ as

$$
\mathbf{y}_{j}=\mathbf{a}_{j} x_{j}+\mathbf{n}_{j}
$$

where $\mathbf{a}_{j}=\left[a_{j j}, \ldots, a_{i j}, \ldots, a_{N j}\right]^{T}$ for $j=1, \ldots, N$ and $i=j, \ldots, N$ and $\mathbf{n}_{j} \sim \mathcal{N}\left(\mathbf{0}, \mathbf{K}_{j}\right)$ in which $\mathbf{K}_{j}=N_{0} \operatorname{diag}\left\{1, \ldots f_{i}\left(\mathbf{R}_{i-1}^{-1}\right)_{j j}, \ldots, f_{N}\left(\mathbf{R}_{N-1}^{-1}\right)_{j j}\right\}=$ $\operatorname{diag}\left\{\sigma_{j j}^{2}, \ldots, \sigma_{i j}^{2}, \ldots, \sigma_{N j}^{2}\right\}$. It can be shown that for the case $\rho_{j i}=\rho$ for all $i \neq j, r_{i} \triangleq\left(\mathbf{R}_{i-1}^{-1}\right)_{j j}=\frac{1+(i-3) \rho}{(1-\rho)(1+(i-2) \rho)}[8]$, independent of $j$. Thus

$$
\sigma_{i j}^{2}=N_{0} \begin{cases}1 & \text { if } i=j \\ f_{i} r_{i} & \text { if } j<i \leq N\end{cases}
$$

where $f_{i}$ follows (15). Note that $r_{i}$ in (21) represents the crosscorrelation impact due to the sum of transmit signals from $U_{i}$.

Let $\mathbf{b}_{j}=\left[\frac{a_{j j}}{\sigma_{j j}^{2}}, \ldots, \frac{a_{i j}}{\sigma_{i j}^{2}}, \ldots, \frac{a_{N j}}{\sigma_{N j}^{2}}\right]^{T}$. Then the desired symbol is detected as

$$
\hat{x}_{j}=\mathbf{b}_{j}^{H} \mathbf{y}_{j}=a_{j} x_{j}+n_{j},
$$

where $a_{j}=\mathbf{b}_{j}^{H} \mathbf{a}_{j}=\sum_{i=j}^{N} \frac{\left|a_{i j}\right|^{2}}{\sigma_{i j}^{2}}$, and $n_{j}=\mathbf{b}_{j}^{H} \mathbf{n}_{j} \sim$ $\mathcal{N}\left(0, \sigma_{j}^{2}\right)$ with $\sigma_{j}^{2}=\sum_{i=j}^{N} \frac{\left|a_{i j}\right|^{2}}{\sigma_{i j}^{2}}$. Thus for BPSK modulation, the detection rule is

$$
\hat{x}_{j}=\operatorname{sgn}\left\{\mathbf{b}_{j}^{H} \mathbf{y}_{j}\right\} .
$$




\section{Detection at Mobile Units for DAF Protocol}

In DAF, MUs decode the overheard symbols, re-encodes, and forwards it to the BS. To re-encode the symbols, a MU is assumed to know all signature waveforms from MUs before it, and this knowledge is used to detect the overheard symbols. At $U_{i}$, matched-filtering is applied to the received signal $y_{j i o}^{D}(t)$ produce the desired symbol $x_{j}$ as

$$
y_{j, u_{i}}=<y_{j i o}^{D}(t), s_{j}(t)>=\sqrt{P_{j j}} h_{j i} x_{j}+n_{j, u_{i}},
$$

where $n_{j, u_{i}} \sim \mathcal{N}\left(0, N_{0}\right)$. Thus for BPSK modulation, the detection rule is

$$
\widehat{x}_{j, u_{i}}=\operatorname{sgn}\left\{<y_{j i o}^{D}(t), s_{j}(t)>\right\} .
$$

Note that only a simple detection is used at MUs.

\section{Performance Analysis}

In this section, we analyze the performance of our proposed scheme that is introduced in Section II. The detection in (23) provides the maximal instantaneous signal-tointerference-plus-noise ratio (SINR) $\gamma_{j}$ corresponding to the desired symbol $x_{j}$ as

$$
\gamma_{j}=\sum_{i=j}^{N} \frac{\left|a_{i j}\right|^{2}}{\sigma_{i j}^{2}}=\frac{P_{j j}\left|h_{j d}\right|^{2}}{N_{0}}+\sum_{i=j+1}^{N} \frac{\widetilde{P}_{i j}\left|h_{i d}\right|^{2}}{f_{i} r_{i} N_{0}},
$$

and (26) will be used to provide BER expressions for DAF and AAF protocols.

\section{A. BER Expression for DAF Protocol}

Let $\beta_{i j}$ for $i=j+1, \ldots, N$ be a binary number representing the detection correctness at $U_{i}$ in detecting $x_{j}$. Then $\beta_{i j}$ is a Bernoulli random variable, which is distributed as

$$
\beta_{i j}= \begin{cases}1 & \text { w.p. } 1-p_{j, u_{i}} \\ 0 & \text { w.p. } p_{j, u_{i}}\end{cases}
$$

where $p_{j, u_{i}}$ is the BER in detection of $x_{j}$ at $U_{i}$. For each information $x_{j}$, the $\beta_{i j}$ 's form a decimal number $S_{j}=$ $\beta_{(j+1) j} \ldots \beta_{i j} \ldots \beta_{N j}$, which represents one of $\left(2^{(N-j)}\right)$ detection states of these $(N-j)$ MUs acting as relays.

Based on (25), the conditional BER of detecting $x_{j}$ at $U_{i}$ can be written as [9]

$$
p_{j, u_{i}}^{h_{j i}}=Q\left(\sqrt{\gamma_{j, u_{i}}}\right)=\frac{1}{\pi} \int_{0}^{\pi / 2} \exp \left(-\frac{\gamma_{j, u_{i}}}{2 \sin ^{2} \theta}\right) d \theta
$$

where $\gamma_{j, u_{i}}=P_{j j}\left|h_{j i}\right|^{2} / N_{0}$ is the instantaneous signal-tonoise ratio (SNR) at $U_{i}$ in detection of $x_{j}$. By averaging (28) with respect to the exponential random variable $\left|h_{j i}\right|^{2}$, the unconditional BER is calculated as

$$
p_{j, u_{i}}=F\left(1+\frac{P_{j j} \sigma_{j i}^{2}}{2 N_{0} \sin ^{2} \theta}\right),
$$

where

$$
F(x(\theta))=\frac{1}{\pi} \int_{0}^{\pi / 2} \frac{1}{x(\theta)} d \theta
$$

Thus the transmit power $\widetilde{P}_{i j}^{D}$ allocated at $U_{i}$ to deliver $x_{j}$ for $i=2, \ldots, N$ and $j=1, \ldots, i-1$ can be expressed as $\widetilde{P}_{i j}^{D}=P_{i j} \beta_{i j}$.

Now, the instantaneous SINR $\gamma_{j}^{D}$ at the destination can be expressed as

$$
\gamma_{j}^{D}=\frac{P_{j j}\left|h_{j d}\right|^{2}}{N_{0}}+\sum_{i=j+1}^{N} \frac{P_{i j} \beta_{i j}\left|h_{i d}\right|^{2}}{r_{i} N_{0}},
$$

where we have used $f_{i}=1$ for DAF. Based on (31), it can be shown that the BER in detecting $x_{j}$ at the BS is [3]

$$
\begin{aligned}
p_{j}^{D} & =\sum_{S_{j}=0}^{2^{(N-j)}-1} F\left(\left(1+\frac{P_{j j} \sigma_{j d}^{2}}{2 N_{0} \sin ^{2} \theta}\right)\right. \\
& \left.\times \prod_{i=j+1}^{N}\left(1+\frac{P_{i j} \beta_{i j} \sigma_{i d}^{2}}{2 r_{i} N_{0} \sin ^{2} \theta}\right)\right) \prod_{i=j+1}^{N} G\left(\beta_{i j}\right)
\end{aligned}
$$

where

$$
G\left(\beta_{i j}\right)= \begin{cases}1-p_{j, u_{i}} & \text { if } \beta_{i j}=1 \\ p_{j, u_{i}} & \text { if } \beta_{i j}=0\end{cases}
$$

and $F($.$) follows (30). From (32), we expect that x_{j}$ will receive a spatial diversity order of $(N-j+1)$.

\section{B. BER Expression for AAF Protocol}

The conditional BER for AAF protocol given the all channel coefficients is

$$
p_{j}^{A,\left\{h_{i d}, h_{j i}\right\}}=Q\left(\sqrt{\gamma_{j}^{A}}\right) .
$$

where $\gamma_{j}^{A}$ follows (26) with $f_{i}$ in (15) for AAF protocol. At the present time, it is difficult to obtain a close-form expression for $f_{i}$ in term of the channel variances $\sigma_{i d}^{2}$ and $\sigma_{j i}^{2}$. Therefore, (34) will be used to provide numerical results to validate our proposed scheme.

\section{NumERicAl Results}

In this section, we perform computer simulations to validate our proposed scheme for both DAF and AAF protocols. In all simulations, the number of MUs $N=4$ and the variance of the noise $N_{0}=1$. We assume all channel variances are 1, i.e., $\sigma_{i d}^{2}=\sigma_{j i}^{2}=1$ for $i=1, \ldots, N$ and $j=1, \ldots i-1$ and the total transmit powers $P_{j}=\sum_{i=j}^{N} P_{i j}$ corresponding to $x_{j}$ are the same for $j$. Furthermore, we assume equal power allocation [3] for $x_{j}$ for $j=1, \ldots, N-1$, i.e.

$$
P_{i j}=\left\{\begin{array}{ll}
\frac{P_{j}}{2} & \text { if } i=j \\
\frac{P_{j}}{2(N-j)} & \text { if } j<i \leq N
\end{array} .\right.
$$

For $x_{N}, P_{N N}=P_{N}$ since it is transmitted directly to the BS. We also assume that the cross-correlation $\rho_{j i}=\rho$ for all $i \neq j$. We use $\rho=0.5$ in our simulations. The MUs are numbered in the reduction order of the distance to the BS; therefore, we expect a diversity order of $4,3,2$, and 1 for $x_{1}, x_{2}, x_{3}$, and $x_{4}$.

Figures 3 and 4 present the analytical and simulation results for DAF protocol and the numerical and simulation results for 


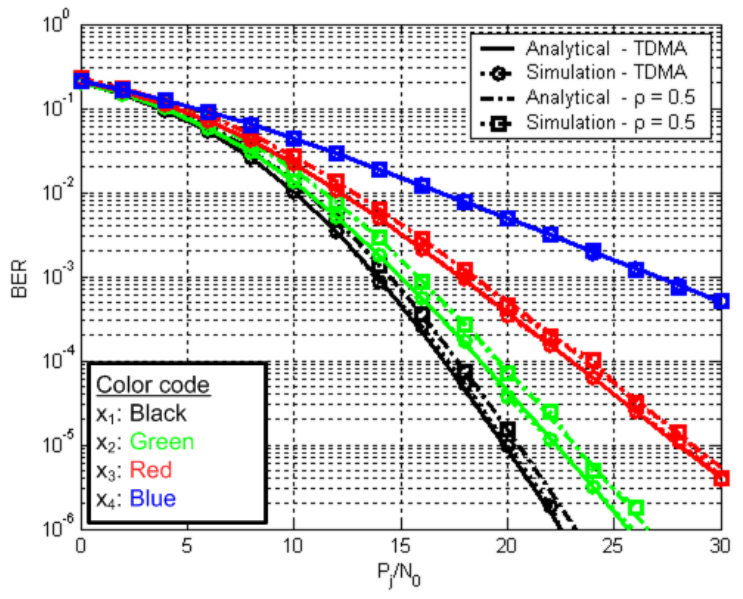

Fig. 3. BER versus SNR performance for DAF protocol - A comparison between the proposed scheme and the conventional TDMA scheme.

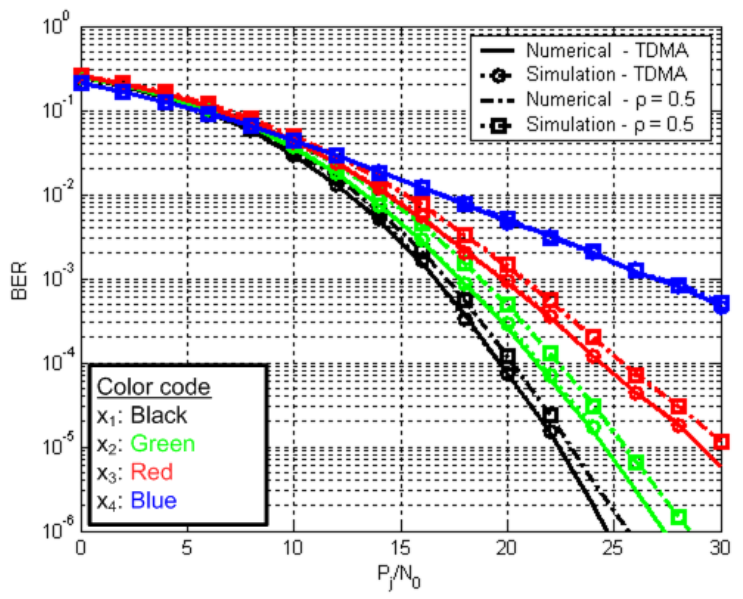

Fig. 4. BER versus SNR performance for AAF protocol - A comparison between the proposed scheme and the conventional TDMA scheme.

AAF protocol, respectively. The figures provide a comparison in term of BER performance between our proposed scheme and the conventional cooperation-based TDMA scheme. In each figure, BER versus SNR $\left(P_{j} / N_{0}\right)$ performance for each information $x_{j}$ is presented. Clearly, our proposed scheme provides the expected diversity orders in both DAF and AAF protocols. In other words, given the use of nonorthogonality, our proposed scheme still achieves full diversity. The diversity orders can be used to reduce the transmit power of distant MUs, given the same QoS with the closer ones. In addition, the figures show that for the case of $\rho=0.5$, the performance gap is less than $1 \mathrm{~dB}$, given the same BER. This is significant because the delay in our proposed scheme is only $(2 N-1)$, a substantial reduction in comparison with the conventional TDMA scheme, which results in a delay of $N(N+1) / 2$.

Figure 5 provides a comparison between $\mathrm{DAF}$ and $\mathrm{AAF}$ protocols. From the figure, DAF protocol outperforms AAF protocol. In particular, there is about $2.5 \mathrm{~dB}$ gap in performance between the two protocols. The figure also shows that the gap reduces as the diversity order decreases. DAF protocol outperforms AAF protocol because of the error propagation

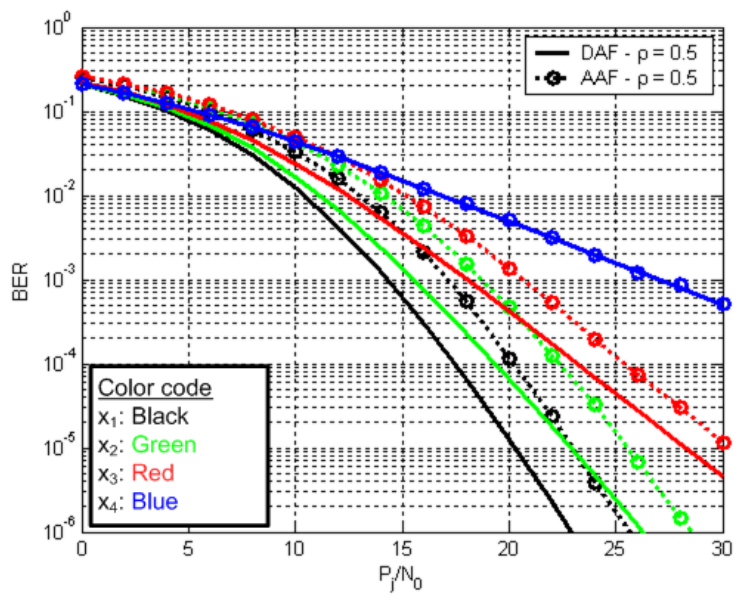

Fig. 5. BER versus SNR performance between DAF and AAF protocol $(\rho=0.5)$.

in AAF protocol. Nevertheless, both DAF and AAF protocols achieve full diversity in our scheme.

\section{CONClusions}

In this paper, we proposed a location-aware cooperationbased scheme using linear network coding, where each MU forms a linear coded version of all symbols it has received previously. The scheme aims to provide to distant MUs higher diversity orders, which can be used to reduce their transmit power. Multiuser detection is used at the BS to decouple the transmit symbols. Both DAF and AAF protocols were considered, performance analysis was presented, and simulations showed that our proposed scheme can achieve a comparable performance but with a substantial reduction in delay in comparison with the conventional cooperation-based TDMA scheme. The delay in our proposed scheme is only $(2 N-1)$ for a network of $N$ MUs while that is $N(N+1) / 2$ in the conventional scheme.

\section{REFERENCES}

[1] J. N. Laneman, D. N. C. Tse, and G. W. Wornell, "Cooperative diversity in wireless networks: efficient protocols and outage behavior," IEEE Trans. Inform. Theory, vol. 50, no. 12, pp. 3062-3080, Dec. 2004.

[2] W. Su, A. K. Sadek, and K. J. R. Liu, "Cooperative communications in wireless networks: performance analysis and optimum power allocation," Wireless Personal Commun., vol. 44, no. 2, pp. 181-217, Jan. 2008.

[3] A. K. Sadek, W. Su, and K. J. R. Liu, "Multinode cooperative communications in wireless networks," IEEE Trans. Signal Process., vol. 55, no. 1 , pp. 341-355, Jan. 2007.

[4] A. Ibrahim, A. K. Sadek, W. Su, and K. J. R. Liu, "Cooperative communications with partial channel state information: when to cooperate?," Proc. IEEE Globecom'05., vol. 5, pp. 3068-3072, Nov. 2005.

[5] A. Ibrahim, A. K. Sadek, W. Su, and K. J. R. Liu, "Cooperative communications with relay selection: when to cooperate and whom to cooperate with?," IEEE Trans. Wireless Commun., vol. 7, no. 7, pp. 2814 2827, July 2008.

[6] J. N. Laneman and G. W. Wornell, "Distributed space-time coded protocols for exploiting cooperative diversity in wireless networks," IEEE Trans. Inform. Theory, vol. 49, pp. 2415-2525, Oct. 2003.

[7] J. G. Proakis, Digital Communications. NewYork, USA: McGraw-Hill, 4th ed., 2001

[8] C. D. Meyer, Matrix Analysis and Applied Linear Algebra. USA: SIAM, 2000.

[9] S. Verdu, Multiuser Detection. New York, USA: Cambridge University Press, 1998. 